\title{
Development and Feasibility of the Misuse, Abuse, and Diversion Drug Event Reporting System (MADDERS ${ }^{\circledR}$ )
}

\author{
Roi Treister, PhD, ${ }^{1,2}$ Jeremiah J. Trudeau, PhD, ${ }^{3}$ Richard Van Inwegen, PhD, ${ }^{2}$ \\ Judith K. Jones, PhD, ${ }^{4,5,6}$ Nathaniel P. Katz, MS, MD ${ }^{2,7}$ \\ ${ }^{1}$ Department of Neurology, Massachusetts General Hospital \& Harvard Medical School, Boston, Massachusetts \\ ${ }^{2}$ Analgesic Solutions, Natick, Massachusetts \\ ${ }^{3}$ Boehringer Ingelheim, Ridgefield, Connecticut \\ ${ }^{4}$ The Degge Group, Fairfax, Virginia \\ ${ }^{5}$ Georgetown University School of Medicine, Washington, District of Columbia \\ ${ }^{6}$ University of Michigan School of Public Health, Ann Arbor, Michigan \\ ${ }^{7}$ Tufts University, Boston, Massachusetts
}

Background and Objectives: Inappropriate use of analgesic drugs has become increasingly pervasive over the past decade. Currently, drug abuse potential is primarily assessed post-marketing; no validated tools are available to assess this potential in phase II and III clinical trials. This paper describes the development and feasibility testing of a Misuse, Abuse, and Diversion Drug Event Reporting System (MADDERS), which aims to identify potentially abuserelated events and classify them according to a recently developed classification scheme, allowing the quantification of these events in clinical trials.

Methods: The system was initially conceived and designed with input from experts and patients, followed by field-testing to assess its feasibility and content validity in both completed and ongoing clinical trials.

Results: The results suggest that MADDERS is a feasible system with initial validity. It showed higher rates of the triggering events in subjects taking medications with known abuse potential than in patients taking medications without abuse potential. Additionally, experts agreed on the classification of most abuse-related events in MADDERS.

Discussion and Conclusions: MADDERS is a new systematic approach to collect information on potentially abuse-related events in clinical trials and classify them. The system has demonstrated feasibility for implementation. Additional research is ongoing to further evaluate its validity.

Scientific Significance: Currently, there are no validated tools to assess drug abuse potential during clinical trials. Because of its ease of implementation, its systematic approach, and its preliminary validation results, MADDERS could provide such a tool for clinical trials. (Am J Addict 2016;25:641-651).

Received April 29, 2016; revised September 14, 2016; accepted October 2, 2016.

Address correspondence to Prof. Katz, MD, MS, Analgesic Solutions, 232 Pond St. Natick, MA 01760.

E-mail: nkatz@analgesicsolutions.com

\section{INTRODUCTION}

Prescription drug abuse, a major public health problem in the United States, is manifested by increased incidence of addiction, emergency department visits, fatal and nonfatal overdoses, accidental pediatric ingestion, injection drug diseases (including HIV and hepatitis), and significant societal costs. $^{1-5}$

Abuse has been defined as any intentional, nontherapeutic use of a drug product or other substance, even once, for the purpose of achieving a desirable psychological or physiological effect. ${ }^{6}$ For the purpose of this paper, the term "abuserelated events" refers to a broader set of behaviors, including behaviors that are confused with abuse (eg, misuse or suiciderelated medication ingestion), important consequences of abuse (eg, overdose), and important concomitants of abuse (eg, tampering), and events that form part of the broader assessment of the abuse potential of a drug (eg, withdrawal).

According to European and Unite States regulatory guidelines for assessing the abuse potential of a drug during its development, sponsors should make every effort to set criteria for assessing abuse, collect complete abuse-related data, and determine the potential risk of abuse-related events. ${ }^{7,8}$ Traditionally, clinical trials have assessed the abuse potential of a drug by capturing reported adverse events (AEs). To capture these events, researchers have used various assessment tools, including algorithms based on AE coding dictionaries (eg, Standardized Medical Dictionary for Regulatory Activities [MedDRA] Queries [SMQ] for substance abuse). These tools, however, fail to identify all relevant types of AEs and do not account for drug accountability discrepancies, that is, differences in the expected amounts of unused medication in a subject's possession at specified periods in a clinical trial. Moreover, the tools lack standardized terminology and have not 
been validated. Indeed, in a comprehensive review, Smith at el al. ${ }^{9}$ concluded that classification of abuse-related events in clinical trials by currently available methods is inadequate and that more-reliable, validated assessments of such events are needed.

In recognition of the need for standardized terminology as a first step in developing a standardized approach to assessment of abuse-related events in clinical trials, the Analgesic, Anesthetic, and Addiction Clinical Trials, Translations, Innovations, Opportunities, and Networks (ACTTION) public-private partnership convened an expert panel to identify the relevant terms and the most appropriate definitions. ${ }^{6}$ The terms and definitions developed by that group were organized into a classification scheme that included six mutually exclusive categories (misuse, abuse, suicide-related, therapeutic error, none of the above, and unknown) plus five supplemental designators (tampering, withdrawal, addiction-related, diversion, and overdose) (Figure 1, adopted with permission from Smith et al. ${ }^{6}$ ).

The ACTTION classification scheme allows for the use of a common terminology among clinical researchers and serves as the foundation for the reliable and accurate classification of abuse-related events. However, the use of such a classification scheme in an actual clinical trial to assess a drug's potential for abuse requires a reliable, systematic, and validated approach that allows the quantification of a drug's potential for abuse or misuse. We therefore developed MADDERS as an extension of the ACTTION scheme. The MADDERS was designed to identify potentially abuse-related events and to promote the collection of the needed information for utilizing the ACTTION classification scheme in clinical trials.

\section{METHODS}

\section{Overview of MADDERS Development Program}

MADDERS was designed as an extension of ACTTION and other existing classification systems for capturing AEs in clinical trials in the arena of prescription drug abuse; it is not intended to be a diagnostic instrument, a scale, or a measure of patient-reported outcomes. The process of MADDERS begins with identifying triggering events, that is, AEs that suggest potential abuse and would trigger the implementation of MADDERS in a clinical trial. In addition to these AEs,

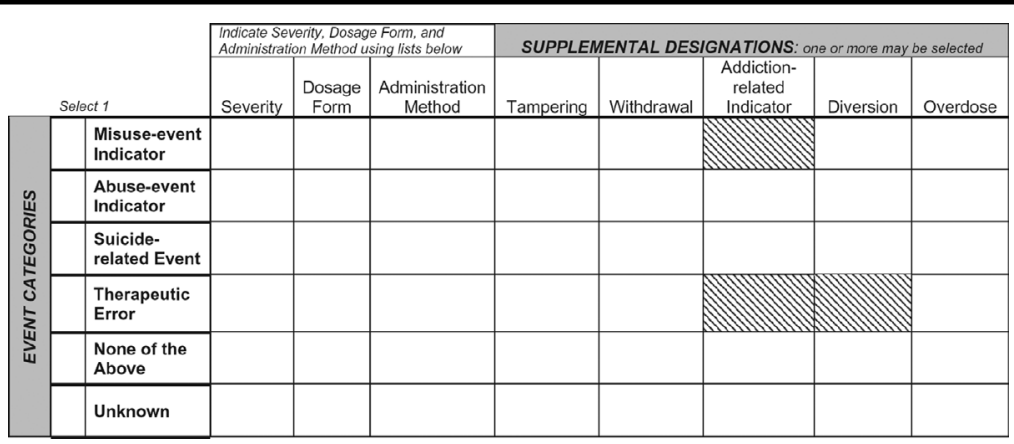

FIGURE 1. Analgesic, Anesthetic, and Addiction Clinical Trials, Translations, Innovations, Opportunities, and Networks (ACTTION) classification scheme for misuse, abuse, and related events. (adapted from Smith et al. ${ }^{9}$; permission obtained from the publisher). For each event, one event category would be chosen; the severity of the event, the dosage form, and the method of administration would be identified; and any supplemental designations providing further information about the event would be selected. Dosage forms: OS: solid designed to be swallowed intact; TM: solid designed for administration through oral mucosa (eg, buccal, sublingual); TD: solid designed for transdermal administration (eg, patch); SS: semisolid designed for transdermal administration (eg, gel, ointment, lotion, cream); OL: liquid designed for administration by swallowing (eg, solutions, suspensions, emulsions); NL: liquid designed for administration intranasally (eg, metered sprays); NA: aerosol designed for administration intranasally; PI: inhalants or sprays designed for pulmonary administration; UNK: unknown. Administration method: Oral; Sublingual; Nasal insufflation (ie, "snorted"); Vaporized and inhaled; Injected; Unknown. Event categories: Misuse-event indicator: Any intentional therapeutic use of a drug product in an inappropriate way. Misuse specifically excludes events that meet the definition of an Abuseevent indicator. Abuse-event indicator: Any intentional, nontherapeutic use of a drug product or substance, even once, for the purpose of achieving a desirable psychological or physiological effect. Suicide-related event: A self-injurious or potentially self-injurious behavior associated with at least some intent to die or that resulted in death. Evidence that the individual intended to kill him/herself, at least to some degree, can be explicit or inferred from the behavior or circumstance. A suicide attempt may or may not result in actual injury (adapted from Posner et al. ${ }^{10}$ ). Therapeutic error: A mistake in a therapeutic regimen. None of the above: Sufficient information exists to determine that none of the previous categories apply. Unknown: Insufficient information exists to determine which category applies. Definitions of supplemental designations: Tampering: The inappropriate manipulation of a drug product. Withdrawal: Symptoms or signs due to the decline in blood concentration of a drug substance (eg, after dose reduction, at the end of a dosing interval, after discontinuing treatment) or due to the administration of an antagonist. Addiction-related indicator: Behavioral, cognitive, and physiological phenomena that may develop after exposure to a substance (typically on a repeated basis), which may include a strong desire to take the drug, difficulties in controlling drug use, persistent drug use despite harmful consequences, intractable and distracting thoughts about the drug, or placing a higher priority on drug use than other activities and obligations. Diversion: Any intentional act that results in transferring a drug product from lawful to unlawful distribution or possession. Diversion can occur with all categories except Therapeutic Error. Overdose: Any act that results in drug exposure exceeding that which is generally recommended or medically accepted. 
triggering events also include drug accountability discrepancies, which are differences in the expected amounts of unused medication in a subject's possession at specified periods in a clinical trial.

Following events identification, another major component of the system is training investigators and other site personnel on all aspects of MADDERS (see Table 1 for some training examples used). For this purpose, a web-based training program was developed.

Our development and initial evaluation of MADDERS included four steps: (i) systematic identification of triggering events; (ii) validation of the list of triggering events; (iii) development of the MADDERS form and cognitive debrief with key stakeholders; and (iv) feasibility assessment of the MADDERS classification process.

The first step in MADDERS is the identification of triggering events. The research team identified a comprehensive list of over 200 unique AE terms related to inappropriate medication use from (i) regulatory guidance documents (U.S. Food and Drug Administration [FDA] and Canadian Health Authority); (ii) SMQ terms for drug dependence; (iii) World Health Organization pharmacovigilance reports on drugs of abuse; (iv) Diagnostic and Statistical Manual of Mental Disorders, Fourth Edition (DSM-IV), listings of signs and symptoms of drug abuse; (v) scientific literature on symptoms of drug abuse and withdrawal; and (vi) databases from cannabinoid and opioid clinical trials. This extended list was reviewed and reduced to approximately 80 terms; items that were unlikely to predict abuse or that were redundant were removed. MedDRA experts who were involved in MedDRA development then reviewed this list. They mapped any nonMedDRA terms to MedDRA Preferred Terms; combined related items into single terms (eg, "drug dependence," "drug dependence antepartum," and "drug dependence postpartum" were collapsed into "drug dependence"); and eliminated duplicate entries that mapped to the same MedDRA Preferred Term. The final list had 60 Preferred Terms (Table 2).

The research team recognized that unlike the case for AEs, the identification of drug accountability discrepancies would depend on the drug formulation, the methods of use (route of administration), and the prescribed amounts. For example, in a trial where subjects are prescribed a weekly patch, one missing patch might be used to trigger evaluation, whereas in a trial where subjects are given a bottle of 120 tablets to last for 30 days, one missing tablet may be an oversensitive criterion. Thus, the group developed a policy whereby the drug discrepancy threshold would need to be defined a priori and justified for each drug and each study.

The second step was to test the validity of the list of 60 triggering events in differentiating drugs with known abuse potential (abused drugs) from drugs without known abuse potential (nonabused drugs). We compared the number of times the terms on our list were reported in the FDA's Adverse Events (FAERS) database for abused and nonabused drugs. Data were retrieved from FAERS from 1998 (when MedDRA coding was implemented) to August 2012.
Drugs were selected for the evaluation of validity of the triggering AE list based on the following principles: (i) only generic drugs were used to avoid biases that might be introduced by brand vs. generic status; (ii) drugs of abuse from multiple pharmacologic classes were used to avoid a list relevant to only one class of abused drugs; (iii) non-abused drugs were also selected from multiple pharmacologic classes that act both centrally and peripherally; (iv) a broad range of indications were used for both abused and non-abused drugs to avoid bias by indication; (v) non-abused drugs that are associated with physical dependence and withdrawal were included in order to avoid imbalance of the AE list towards withdrawal.

Additionally, for the statistical benefit of large sample size, only drugs with more than 1,000 reported AEs were included in the analysis. The representative abused drugs evaluated were alprazolam, dextromethorphan, diazepam, hydrocodone, methylphenidate, and oxycodone; the nonabused drugs were duloxetine, sodium valproate, aripiprazole, and metoprolol.

To examine the utility of each AE Preferred Term in the FAERS database, we categorized it as having positive, neutral, or negative value by using an artificial cutoff of $\pm .1 \%$ difference in the term's frequency between abused and nonabused drugs (ie, a difference of more than $.1 \%$ was classified as positive; a difference of less than $-.1 \%$ was considered negative; and a difference in the range of -.1 to .1 was neutral). Because this is the first-ever development of such a system for assessing abuse potential in clinical trials, we had no a priori thresholds, so the $.1 \%$ cutoff was based on the data at hand.

In the third step, to capture the additional information needed to classify the triggering events according to the abuserelated terms, definitions, and classification scheme developed by the ACTTION panel, we designed the MADDERS form. A key element in capturing this information is real-time (or near real-time) interviews with subjects. These timely interviews will allow researchers to collect the most accurate and least biased information about the triggering event immediately after it was identified during the clinical trial.

After discussing with three drug-abuse experts what information was needed to classify events into each abuserelated term, we generated our first MADDERS form (version 1). Given the wide range of potential triggering events, we decided that the form should be interview-guided that will be completed by clinicians rather than subjects. The form guides the interviewer about the information that should to be collected, without providing exact text to read to the subjects. (ie, structured interview). In response to the experts' review of the form (see next paragraph), we made minor modifications to it.

To assess the feasibility of the interview process for completing the MADDERS form, we asked four site clinicians involved in recently completed analgesic clinical trials to conduct interviews with 18 trial subjects and to complete the MADDERS form as if this were done at the trial they had recently completed. We collected input from both clinicians 
Event categories

Misuse: Any intentional therapeutic use of a drug product in an inappropriate way.

Abuse: Any intentional, nontherapeutic use of a drug product or substance, even once, for the purpose of achieving a desirable psychological or physiological effect.

Suicide-related: A self-injurious or potentially self-injurious behavior associated with at least some intent to die or that resulted in death.

Therapeutic error: A mistake in a therapeutic regimen.

None of the above: Sufficient information exists to determine that none of the previous categories apply.

Unknown: Insufficient information exists to determine which category applies.
A cancer patient presents to the clinic with two vials missing. His wife indicates that he has been using somewhat more medication than prescribed in order to get better pain relief, which has been working for him, but he has been increasingly tired.

A patient in a blinded clinical trial admits saving up his study medication during the week so he can use it at parties to "get in the mood." He reports no untoward medical events associated with this use pattern.

A patient in a clinical trial was difficult to arouse and was taken to the ER. Upon awakening, she indicated that her pain was increasingly out of control and she had lost hope for a cure. She also acknowledged being increasingly despondent and having taken all her remaining prescribed opioid pills since she did not care whether she lived or died.

A patient in a clinical trial had one missing tablet. He reported that he had taken two tablets instead of one by accident.

A patient in a clinical trial reported that he was euphoric/elated due to his diminished pain.

A patient in an open-label study of opioids did not return for his routine monthly clinic visit 1 month into the study and despite repeated efforts could not be located for follow-up.

\section{Supplemental designations}

Tampering: Inappropriate manipulation of a drug product.

Withdrawal: Symptoms or signs due to the decline in blood concentration of a drug substance.

Addiction-related indicator: Behavioral, cognitive, and physiological phenomena that may develop after exposure to a substance (typically on a repeated basis) and which may include a strong desire to take the drug, difficulties in controlling drug use, persistent drug use despite harmful consequences, intractable and distracting thoughts about the drug, or placing a higher priority on drug use than on other activities and obligations.

Diversion: Any intentional act that results in transferring a drug product from lawful to unlawful distribution or possession.

Overdose: Any act that results in drug exposure exceeding that which is generally recommended or medically accepted.
A 47-year-old man with multiple myeloma in hospice was in a clinical trial of a cannabinoid for nausea. In the course of routine chemotherapy, he developed moderate oropharyngeal mucositis and was unable to swallow. To prevent withdrawal and maintain pain relief, the clinicians crushed the tablet and put it down the G-tube. The patient was very drowsy for a few hours after the first dose but recovered.

During a routine clinic visit, a patient in a blinded clinical trial reported that she had run out of her medications 2 weeks earlier and that a day after running out, she felt sick, being jittery and nauseated. This feeling lasted a few days but then returned to normal.

A 16-year-old boy in an open-label safety study of an opioid for chronic pain did not show up for a routine clinic visit, and the family indicated that he had been in and out of the ER over the past few weeks for taking too much of his medication. He had also been missing school, and there was missing liquor in the liquor cabinet. In recent months, he had been smoking increasing amounts of marijuana and cigarettes and drinking with new friends.

A 38-year-old woman patient had 20 tablets missing at a routine clinic visit. Upon questioning, she acknowledged sharing the medication with her sister, who was having terrible insomnia after the death of her spouse.

A 61-year-old cancer patient normally taking 4 pills twice a day took 10 one evening in order to have better pain control. He was somnolent and dizzy for several hours, then recovered. 
and subjects about the structured interview through a paperbased survey. The subjects were asked to answer the following questions on a $0-10$ numerical rating scale: How well did the process work $(0=$ not well at all; $10=$ extremely well $)$ ? How much difficulty did you have understanding the questions $(0=$ no difficulty, $10=$ extreme difficulty)? How truthful would you be in answering questions about your study medication use $(0=$ always truthful, $10=$ never truthful $)$ ? Clinicians were asked about the feasibility of the MADDERS form and the interview-guided process.

The fourth and final step was to evaluate the feasibility of using the classification process in a clinical trial. We asked three experts in prescription drug abuse who had completed the MADDERS training to independently classify 10 mock cases. The mock cases were presented to the experts as subject narratives based on data that could have been captured on the MADDERS form in an actual clinical trial.

\section{Statistical Analysis}

To assess differences in the numbers of $\mathrm{AE}$ events identified in the FAERS database between abused and nonabused drugs, we used a paired $t$-test. The Fleiss kappa test was used to assess agreement between experts' classifications of the 10 mock cases. We considered $p$ values significant if they were below .05 .

\section{RESULTS}

During the period that the FAERS database covered (1998 to August 2012), there were 58,973 reported AEs (whether the $\mathrm{AE}$ was what we defined as a triggering event or not) for abused drugs and 51,831 for nonabused drugs (Table 2). The mean number of events for each of the top 20 terms reported for abused drugs (out of the list of 60 triggering events) was significantly higher (paired $t$-test, $p<.001$ ) for abused drugs $(1,628$, or $2.8 \%$ of total AEs) than for nonabused drugs $(725$, or $1.4 \%$ of total AEs). Among all 60 terms in Table 2, 19 (32\%) were considered positive terms (ie, the AE occurred more frequently in abused than in nonabused drugs); 25 (42\%) were considered neutral; and 16 (27\%) were negative (ie, the $\mathrm{AE}$ occurred less frequently in abused than in nonabused drugs). There was a significantly higher $(p=.038)$ cumulative percentage of AEs among abused drugs (68.4\%) than among nonabused drugs (41.9\%). Figure 2 shows some important distributions of these AEs. The top panel shows that among the top 20 Preferred Terms reported for abused drugs, overdose was the most frequent $\mathrm{AE}$ (5,573 reports, or $9.5 \%$ of total AEs). The bottom panel presents the 20 AEs that demonstrated the highest differences (in percentage) in occurrence for abused drugs and nonabused drugs.

The results of the survey about the feasibility of the interview-guided MADDERS form were instructive. The subjects responded positively to the process (mean score 9.9 on $0-10$ scale; $0=$ not well at all, $10=$ extremely well). They indicated that they had little difficulty understanding the questions (mean score .3 on $0-10$ scale; $0=$ no difficulty, $10=$ extreme difficulty), with little explanation needed for most questions. Most subjects indicated that they would answer questions about their study medication use truthfully, although a few indicated they might not be completely honest (mean score 1.8 on $0-10$ scale; $0=$ always truthful, $10=$ never truthful) or that others might lie about abuse and tampering.

The four site clinicians who were surveyed about the feasibility of the MADDERS form and interview process considered the questions on the MADDERS form clear; they thought that the questions gathered sufficient and appropriate information, and the clinicians reported that the subjects' responses were accurate and honest overall.

Finally, the results for assessing the feasibility of using MADDERS to classify triggering events in clinical trials were highly consistent. Among the three experts in MADDERS who classified 10 mock cases prepared according to MADDERS forms and guidelines, there was $90 \%$ agreement (9/10 narratives). Fleiss kappa test for agreement between the experts' classifications was $.881(p<.001)$.

\section{DISCUSSION}

We have described the development and initial feasibility testing of MADDERS, a system that goes beyond the ACTION classification scheme and enables researchers to characterize abuse-related events in clinical trials. The list of MedDRA Preferred Terms used to identify triggering events in clinical trials has demonstrated usability, with a manageable number of identified events. Subsequently, the differences between the distribution of identified triggering events in abused drugs and those in nonabused drugs was demonstrated using the FAERS database. Experts in addiction and abuse, site clinicians, and subjects in clinical trials supported the content validity of MADDERS.

Identifying specific events in clinical trials requires careful, thorough, and standardized assessment and classification. For instance, Posner et al. ${ }^{10,11}$ have developed a method to categorize suicidal events occurring in antidepressant clinical trials. The strength of this suicide classification system is its ability to comprehensively identify suicidal events while limiting the over identification of suicidal behavior. MADDERS uses a similar systematic approach to classify potentially abuse-related events in clinical trials. It is designed as an extension of existing systems for capturing AEs in the arena of prescription drug abuse and is not intended to be a diagnostic instrument, a scale, or a measure of patient-reported outcomes. Nonetheless, additional information about the measurement properties of MADDERS would help refine the system and improve its effectiveness. For example, using MADDERS in clinical trials that compare a known potentially abused medication with a nonabused medication would help explore the system's criterion validity. Similarly, determining whether the system can detect changes in inappropriate uses of medication over time, particularly in a trial of a treatment 


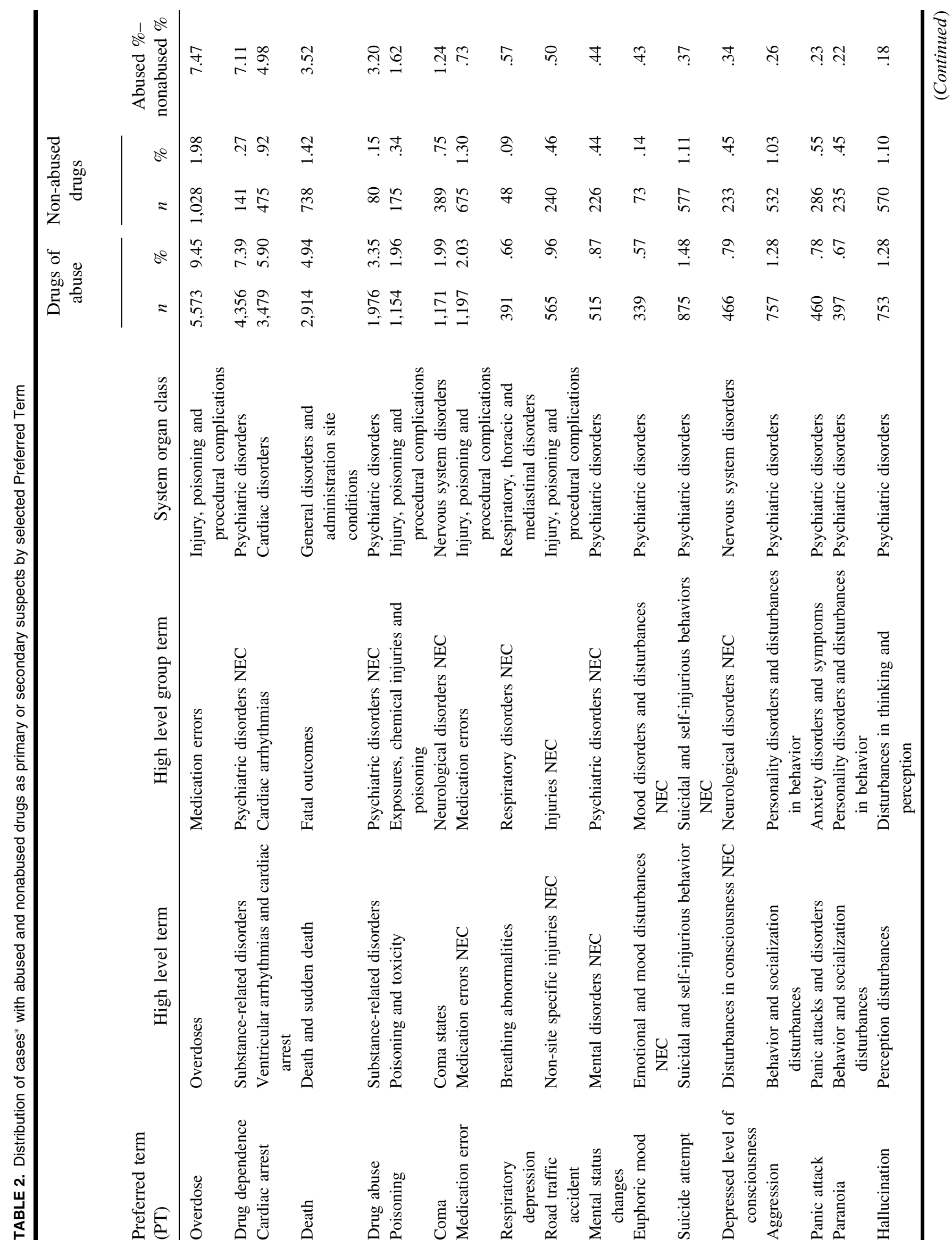




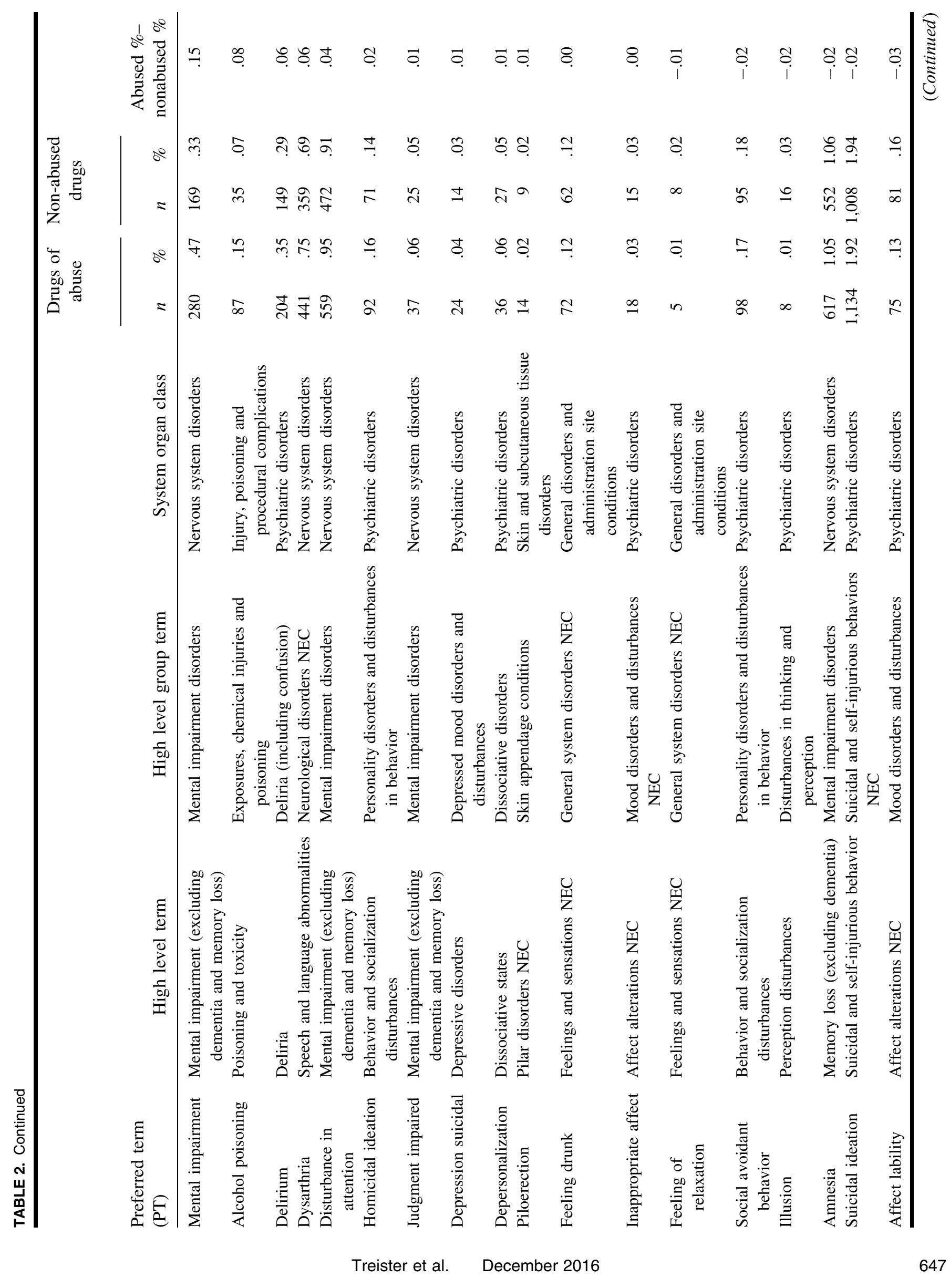




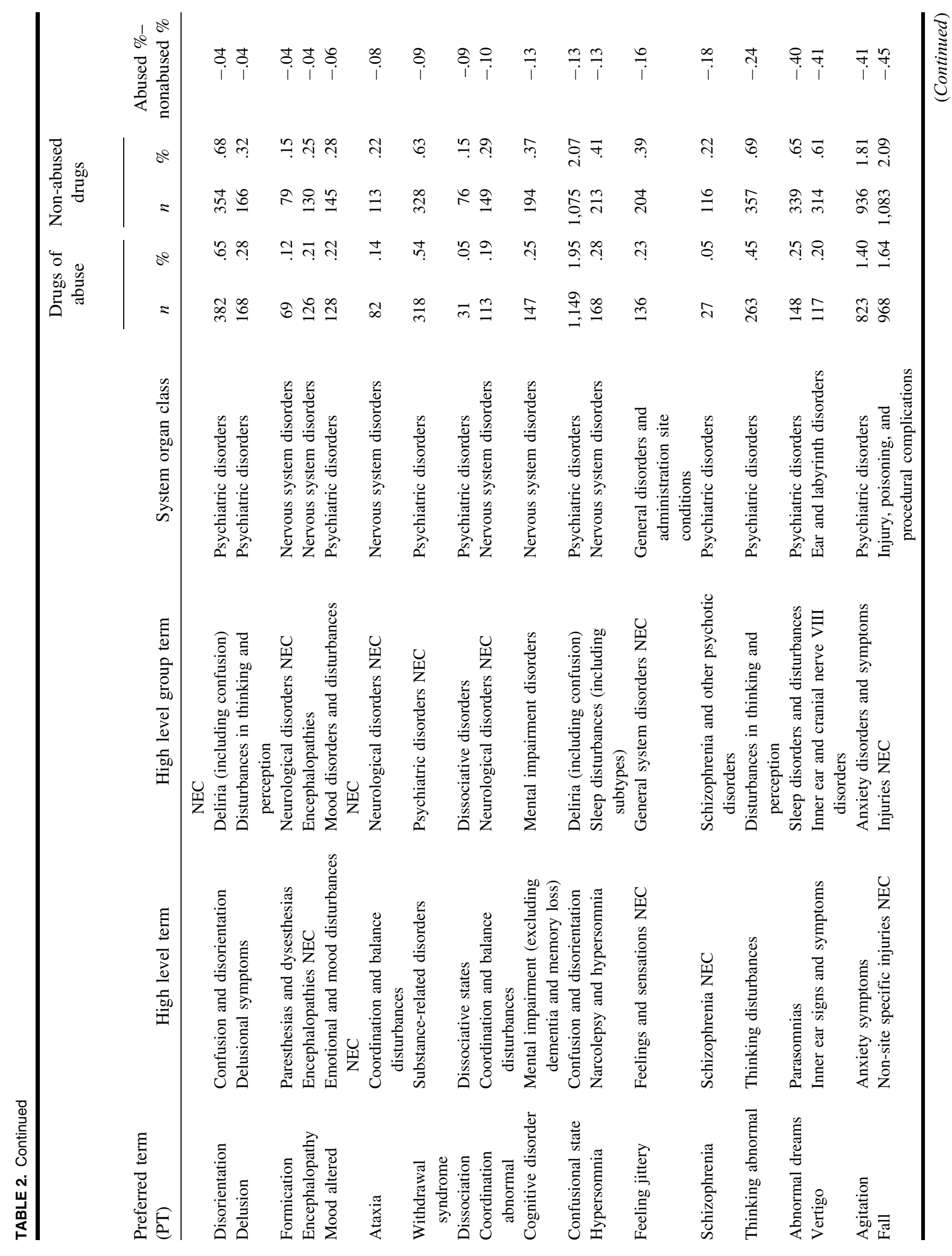




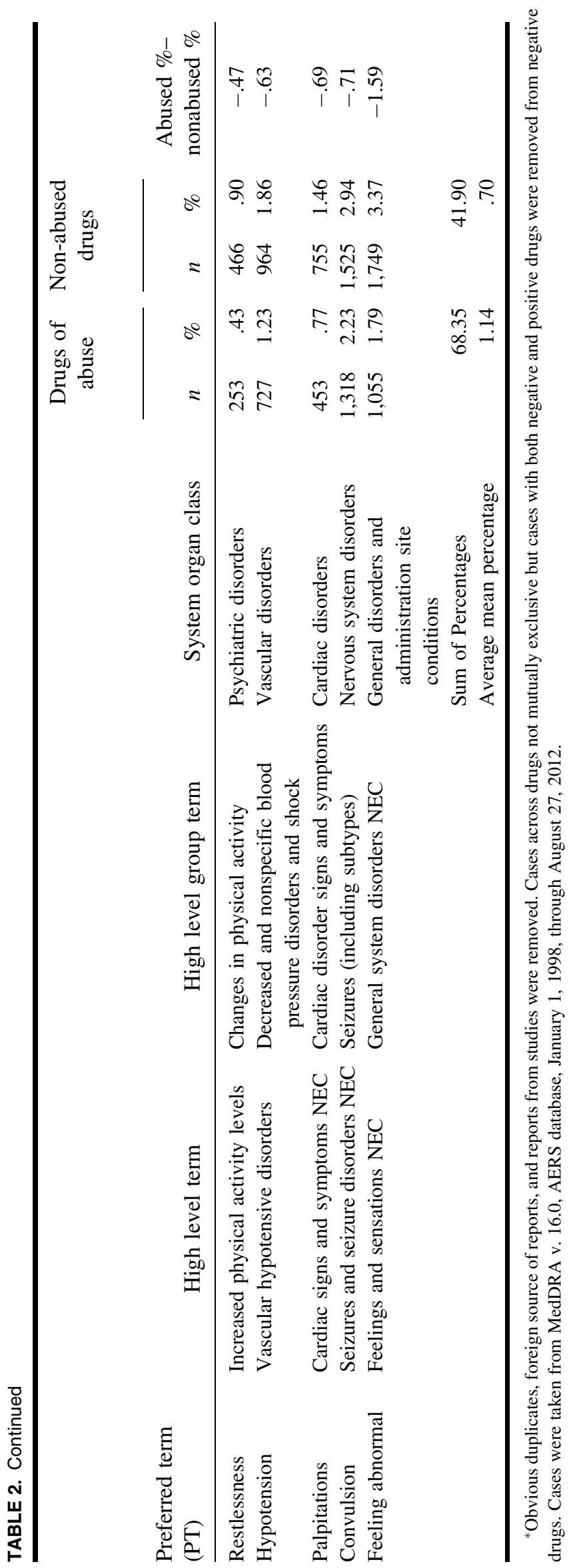

known to reduce abuse, would further refine MADDERS. Our analysis of the FAERS database demonstrates the validity of the list of $\mathrm{AE}$ terms in discriminating abused prescription drugs from nonabused ones. However, among our 60 Preferred Terms, only about one-third differentiated the two groups. Additional validity testing of MADDERS with data sets from clinical trials of compounds with known abuse potential is ongoing. Collecting additional data will allow the opportunity to refine and optimize the triggering event identification process. This would increase sensitivity and specificity and will establish "normative data" that will facilitate comparison across studies/drugs.

The system was designed to obtain more thorough information on intent, behavior, and other contextual factors associated with potentially abuse-related events by training investigators to (i) play an active role in identifying triggering events and (ii) obtain additional information from subjects via guided interviews as soon as possible after an event occurs. This approach provides more comprehensive information, which is expected to lead to greater accuracy in the classification of potential abuse-related events.

However, a few limitations are worth mentioning. First, because MADDERS relies on subjects' reports during an interview with a clinician, candor might be an issue. Nevertheless, asking always yields more information than does failing to ask, and the standardized, systematic nature of an interview based on the MADDERS form reduces bias in the assessments. Second, the data we have presented demonstrate the system's potential feasibility and initial validity, and additional testing in trials of drugs with known abuse liability is ongoing to further validate MADDERS. Third, the abuserelated data on the specialized population of subjects who have given their consent and who are included and monitored in clinical trials might not apply to the general population. However, MADDERS is not intended to substitute for current methods of assessing abuse liability. By collecting relevant information systematically, this approach will allow the interpretation and use of data that are already collected in clinical trials (ie, AEs and drug accountability discrepancies) for the assessment of the abuse potential of a drug. Fourth, in this initial retrospective validation study, drug discrepancies could not be assessed. Ongoing prospective studies have been providing valuable information confirming the need for a policy whereby the drug discrepancy threshold would be defined a priori and justified for each drug and each study.

In the development of any drug, research on its potential for abuse must strike a fine balance between good sensitivity and over identification of triggering events. A lack of sensitivity could fail to uncover potential problems with a drug, whereas over identification of AEs could curtail, for example, the development of an effective pain medication. Future research is needed to refine triggering-event identification and quantification to better balance sensitivity and over classification.

The necessary steps in assessing abuse potential during various stages of drug development, including in vitro 


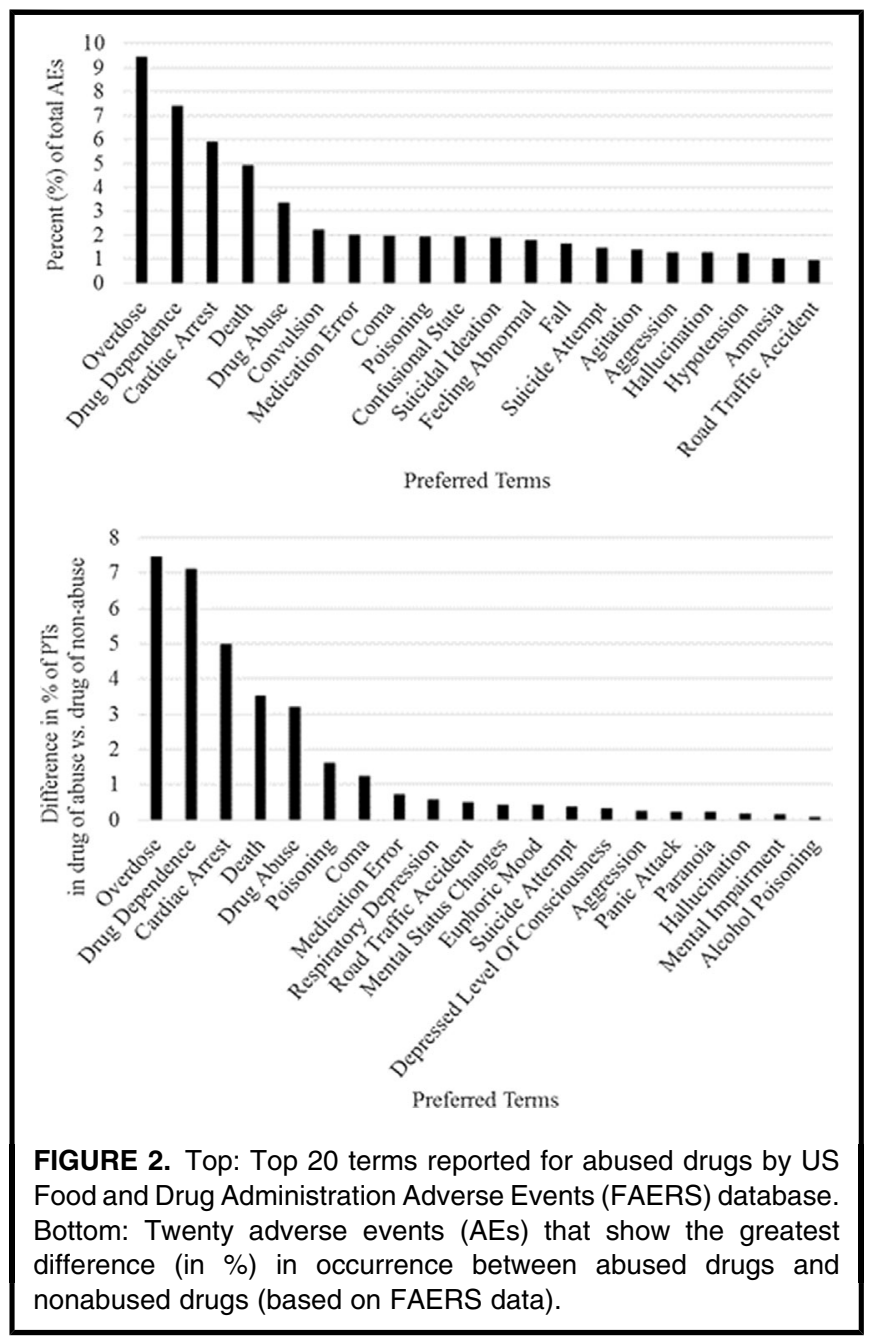

pharmacology, in vitro formulation tests, preclinical studies of abuse potential, phase I pharmacokinetic studies, and phase I human abuse liability studies, are well established and commonly used. ${ }^{12}$ Ironically, there are no standardized, systematic methods for quantifying abuse-related events in phase II and phase III clinical trials where drugs are actually given to the target population for the target indication. ${ }^{9,13-16}$ In their recent review on the inappropriate use of medication, Smith et al. ${ }^{9}$ conclude that the available instruments are not well suited for assessing the inappropriate use of medication within clinical trials. The researchers add that further effort is needed to develop reliable and valid instruments to conduct such assessments. In the face of concerns about abuse of, and addiction to, certain classes of analgesic medications (eg, opioids), as well as opposite concerns that people with chronic pain conditions will not receive adequate pain treatment, it is crucial to accurately estimate the incidence of misuse, abuse, and related events in clinical trials to properly balance the risks and benefits of these medications. MADDERS has been developed to systematize the collection of essential information so that potentially abuse-related events can be classified and their frequency assessed.
To date, the use of MADDERS to identify triggering events has content validity. We base this observation on literature reviews and expert input, as well as data indicating higher incidences of triggering events for abused drugs than for nonabused drugs. Experts have indicated that the MADDERS forms used are practical and content-valid for assessing abuserelated events. Field testing has shown MADDERS to be feasible for the experts who classified the mock cases, for trial subjects, and for site clinicians. Based on the current results, a refined version of MADDERS has been implemented in multicenter trials. To our knowledge, it is the only system currently available for classifying and quantifying abuserelated events in clinical trials.

We acknowledge valuable feedback about MADDERS and its validation during discussions at an ACTTION meeting, "Recommendations for Quantifying Abuse-Related Events in Clinical Trials," held June 27-28, 2013, in Washington, DC. In addition, we thank the following experts for content review and adjudications: Drs. Roger Weiss (Harvard Medical School), Richard Dart (Rocky Mountain Poison and Drug Center), Jennifer Sharpe Potter (UT Health Science Center San Antonio), and Ajay Wasan (University of Pittsburg Medical School). Review and input from Drs. Gil Sperings (MedVadis), Steven Wright (Analgesic Solutions), and Steve Passik (Vanderbilt Medical School) are also gratefully acknowledged. We also thank GW Pharmaceuticals for the use of their data and continued support.

\section{Declaration of Interest}

RT, JJT, RVI, and NPK were all employed in Analgesic Solutions, the developer of MADDERS.

\section{REFERENCES}

1. Denisco RA, Chandler RK, Compton WM. Addressing the intersecting problems of opioid misuse and chronic pain treatment. Exp Clin Psychopharmacol. 2008;16:417-428.

2. Hall AJ, Logan JE, Toblin RL, et al. Patterns of abuse among unintentional pharmaceutical overdose fatalities. JAMA. 2008;300: 2613-2620.

3. Manchikanti L, Helm S 2nd, Fellows B, et al. Opioid epidemic in the United States. Pain Physician. 2012;15:ES9-38.

4. Substance Abuse and Mental Health Services Administration. Results from the 2010 National Survey on Drug Use and Health, vol. 1. Summary of national findings. Rockville, MD: Substance Abuse and Mental Health Services Administration; 2011.

5. Substance Abuse and Mental Health Services Administration. Treatment episode data set (TEDS). Highlights-2007. National Admissions to Substance Abuse Treatment Services. Rockville, MD: Substance Abuse and Mental Health Services Administration, 2009. Office of Applied Studies, DASIS Series S-45, DHHS Publication SMA 09-4360.

6. Smith SM, Dart RC, Katz NP, et al. Analgesic, Anesthetic, and Addiction Clinical Trials, Translations, Innovations, Opportunities, and Networks (ACTTION) public-private partnership. Classification and definition of misuse, abuse, and related events in clinical trials: ACTTION systematic review and recommendations. Pain. 2013;154:2287-2296. 
7. European Medicines Agency. Guideline on the clinical development of medicinal products indented for the treatment of pain (May 30, 2013). http://www.ema.europa.eu/docs/en_GB/document_library/ Scientific_guideline/2013/05/WC500143769.pdf

8. U.S. Department of Health and Human Services, Food and Drug Administration, Center for Drug Evaluation and Research. Guidance for industry: Assessment of abuse potential of drugs. Silver Spring, MD: U.S. Department of Health and Human Services; 2010. http://www.fda.gov/downloads/Drugs/ GuidanceComplianceRegulatoryInformation/Guidances/UCM198650.pdf/

9. Smith SM, Paillard F, McKeown A, et al. Instruments to identify prescription medication misuse, abuse, and related events in clinical trials: An ACTTION systematic review. J Pain. 2015;16:389-411.

10. Posner K, Oquendo MA, Gould M, et al. Columbia Classification Algorithm of Suicide Assessment (C-CASA): Classification of suicidal events in the FDA's pediatric suicidal risk analysis of antidepressants. Am J Psychiatry. 2007;64:1035-1043.

11. Posner K, Brown GK, Stanley B, et al. The Columbia-Suicide Severity Rating Scale: Initial validity and internal consistency findings from three multisite studies with adolescents and adults. Am J Psychiatry. 2011;168:1266-1277.

12. Katz N. Abuse-deterrent opioid formulations: Are they a pipe dream? Curr Rheumatol Rep 2008;10:11-18.

13. Becker WC, Fraenkel L, Edelman EJ, et al. Instruments to assess patientreported safety, efficacy or misuse of current opioid therapy for chronic pain: A systematic review. Pain. 2013;154:905-916.

14. Chou R, Fanciullo GJ, Fine PG, et al. Opioids for chronic noncancer pain: Prediction and identification of aberrant drug-related behaviors: A review of the evidence for an American Pain Society and American Academy of Pain Medicine clinical practice guideline. J Pain. 2009; 10:131-146.

15. O'Connor AB, Turk DC, Dworkin RH, et al. Abuse liability measures for use in analgesic clinical trials in patients with pain: IMMPACT recommendations. Pain. 2013;154:2324-2334.

16. Passik SD, Kirsh KL, Casper D. Addiction-related assessment tools and pain management: Instruments for screening, treatment planning, and monitoring compliance. Pain Med. 2008;9:S145-166. 